\title{
Factors associated with completion of bowel cancer screening and the potential effects of simplifying the screening test algorithm
}

\author{
Benjamin Kearns ${ }^{*}, 1$, Sophie Whyte ${ }^{1}$, Helen E Seaman ${ }^{2}$, Julia Snowball ${ }^{2}$, Stephen P Halloran ${ }^{2,3}$, Piers Butler ${ }^{2}$, \\ Julietta Patnick ${ }^{4}$, Claire Nickerson ${ }^{4}$ and Jim Chilcott ${ }^{1}$ \\ ${ }^{1}$ School of Health and Related Research, University of Sheffield, Sheffield S1 4DA, UK; ${ }^{2}$ NHS Bowel Cancer Screening Southern \\ Programme Hub, Guildford, Surrey GU2 7YS, UK; ${ }^{3}$ Department of Biochemical Sciences, University of Surrey, Guildford, Surrey \\ GU2 7XH, UK and ${ }^{4}$ Public Health England, Sheffield, S10 3TH, UK
}

\begin{abstract}
Background: The primary colorectal cancer screening test in England is a guaiac faecal occult blood test (gFOBt). The NHS Bowel Cancer Screening Programme (BCSP) interprets tests on six samples on up to three test kits to determine a definitive positive or negative result. However, the test algorithm fails to achieve a definitive result for a significant number of participants because they do not comply with the programme requirements. This study identifies factors associated with failed compliance and modifications to the screening algorithm that will improve the clinical effectiveness of the screening programme.
\end{abstract}

Methods: The BCSP Southern Hub data for screening episodes started in 2006-2012 were analysed for participants aged 60-69 years. The variables included age, sex, level of deprivation, gFOBt results and clinical outcome.

Results: The data set included 1409335 screening episodes; 95.08\% of participants had a definitively normal result on kit 1 (no positive spots). Among participants asked to complete a second or third gFOBt, $5.10 \%$ and $4.65 \%$, respectively, failed to return a valid kit. Among participants referred for follow up, $13.80 \%$ did not comply. Older age was associated with compliance at repeat testing, but non-compliance at follow up. Increasing levels of deprivation were associated with non-compliance at repeat testing and follow up. Modelling a reduction in the threshold for immediate referral led to a small increase in completion of the screening pathway.

Conclusions: Reducing the number of positive spots required on the first gFOBt kit for referral for follow-up and targeted measures to improve compliance with follow-up may improve completion of the screening pathway.

Bowel cancer screening and compliance. Colorectal cancer (CRC) is a common form of cancer, with high mortality (Cancer Research UK, 2013). Colorectal cancer is the second most common cause of cancer-related mortality in a number of countries, including the United Kingdom, USA, Japan, Australia, Canada and Germany (Honein-AbouHaidar et al, 2014). Several randomised controlled trials (RCTs) have demonstrated a reduction in CRC mortality with screening (Mandel et al, 1993; Kewenter et al, 1994; Kronborg et al, 1996; Hardcastle et al, 1996) - whereas CRC-related mortality was reduced by $16 \%$ among people offered guaiac faecal occult blood test (gFOBt) screening, it was reduced by $25 \%$ among people who attended at least one round of screening (Hewitson et al, 2008), demonstrating the importance of uptake.

Many studies have investigated the factors associated with CRC screening uptake, along with the barriers to screening (Brouse et al, 2003; Beydoun and Beydoun, 2008; Robb et al, 2008; von Wagner et al, 2009; Von Wagner et al, 2009; Weller and Campbell, 2009; Pornet et al, 2010; Aubin-Auger et al, 2011; Moss et al, 2012; Honein-AbouHaidar et al, 2014; Sicsic and Franc, 2014;

*Correspondence: B Kearns; E-mail: b.kearns@sheffield.ac.uk

Received 21 August 2015; revised 27 November 2015; accepted 4 December 2015; published online 14 January 2016

(c) 2016 Cancer Research UK. All rights reserved 0007-0920/16 
Smith et al, 2014) but there has been little research into the factors that are associated with completion of a screening episode among participants who return a first gFOBt. Compliance with a screening test result has an important impact on clinical outcomes (Gupta, 2008).

Bowel cancer screening in England. The National Health Service (NHS) Bowel Cancer Screening Programme (BCSP) was introduced in England in July 2006 after evaluation of a pilot study in England and Scotland (UK Colorectal Cancer Screening Pilot Group, 2004). The BCSP achieved a nationwide coverage for 60- to 69-year olds in 2010 (NHS Bowel Cancer Screening Programme, 2011) and for 60- to 74-year olds in 2015 (Bowel Cancer Screening Southern Programme Hub, 2015). The BCSP currently offers biennial screening with the gFOBt to all eligible people. Uptake of screening, defined as the proportion of invited subjects with a normal (negative) or abnormal (positive) gFOBt result, varies according to age, sex, socioeconomic status and screening history (Robb et al, 2008; von Wagner et al, 2009; Von Wagner et al, 2009; Pornet et al, 2010; Moss et al, 2012; Smith et al, 2014; Lo et al, 2014b). Participants with a definitive abnormal gFOBt result are invited to a specialist screening practitioner (SSP) clinic to discuss the test result and the need for further investigation (usually by colonoscopy). Completion of a screening episode is defined as achieving a definitive gFOBt result and, for gFOBt-positive participants, receiving a follow-up clinical investigation.

The current screening algorithm in England. The algorithm used by the BSCP to reach a definitive gFOBt result of normal or abnormal is unique to the programme in England. It is based on an algorithm developed for the Nottingham RCT (Hardcastle et al, 1996), which had the explicit aim of reducing the number of false positives (Robinson et al, 1995; Hardcastle et al, 1996). Each gFOBt kit has three pairs of windows. Two small faecal samples from three separate stools are applied by the screening participant to each of the windows in turn, and any of the six windows or 'spots' can test positive for the possible presence of blood in the sample when tested in the laboratory. Participants with a positive spot count of 5-6 ('strong positive') are referred directly to an SSP clinic for further investigation. On the first completed test kit, a positive spot count of 1-4 is considered to provide insufficient evidence of a bowel lesion and is designated an 'unclear' result. A participant with an 'unclear' result is asked to complete a second and, dependent on the outcome of this kit, a third repeat gFOBt kit to enable the programme to reach a definitive test result. One or more positive spots on a valid second kit (or on a third valid kit if all spots were negative on the second kit) provide a 'weak positive' definitive test result and the participant is referred for clinical investigation. The screening algorithm allows for up to three valid kits within a single screening episode, but the total number of kits returned may be greater due to kits that are spoilt (completed incorrectly by the participant) or technical fails (a problem encountered during laboratory analysis), which do not count as valid kits. The current referral algorithm for screening with gFOBt in England is illustrated in Figure 1.

Alternative screening algorithms. Other countries use different gFOBt referral algorithms. For example, different thresholds for direct referral may be used (Benson et al, 2012; Altobelli et al, 2014), or a mixture of screening modalities may be employed (Fraser et al, 2012). Most programmes outside the UK using a gFOBt refer participants with any positive spots on their first (and only) valid kit (Benson et al, 2012), which is consistent with the referral algorithm used in the three (non-English) RCTs of gFOBt screening (Mandel et al, 1993; Kewenter et al, 1994; Kronborg et al, 1996).

Study aims. This study had two main aims. The first aim was to identify the factors that are associated with the completion of a screening episode. The second aim was to examine the relationship between the result of each gFOBt kit completed during a screening episode and completion of the screening episode (to a definitive gFOBT test result or, for gFOBt-positive participants, to colonoscopy or other diagnostic test), and to model the possible effects on completion if the threshold for immediate referral to follow-up were lowered from five to three or four positive spots for the first gFOBt kit.

\section{MATERIALS AND METHODS}

Data were provided by the BCSP Southern Hub for first screening episodes that started between October 2006 and October 2012 (inclusive) among 60- to 69-year olds who had returned at least one valid gFOBt during the study period. The Southern Hub serves a population of over 14 million people in the south of England (Bowel Cancer Screening Southern Programme Hub, 2014; excluding London).

The study was designed to investigate improvements to the BCSP screening algorithm using anonymised data. The study was approved by the BCSP Research Committee and Ethical Committee approval was not required.

The data set included participant age, sex and deprivation score and comprehensive gFOBt spot results, test completion dates and clinical outcomes. The Index of Multiple Deprivation (IMD) score was derived from the participant's area of residence (overall IMD 2007) (Communities and Neigbourhoods, 2010). The IMD is measured on a scale of 0 to 100 , with larger values denoting areas with greater deprivation. During the study period, the process of extension to 70- to 74-year olds was incomplete. Participants aged 70 years and above were therefore excluded from the analysis as this age group was not representative of the population that was offered screening.

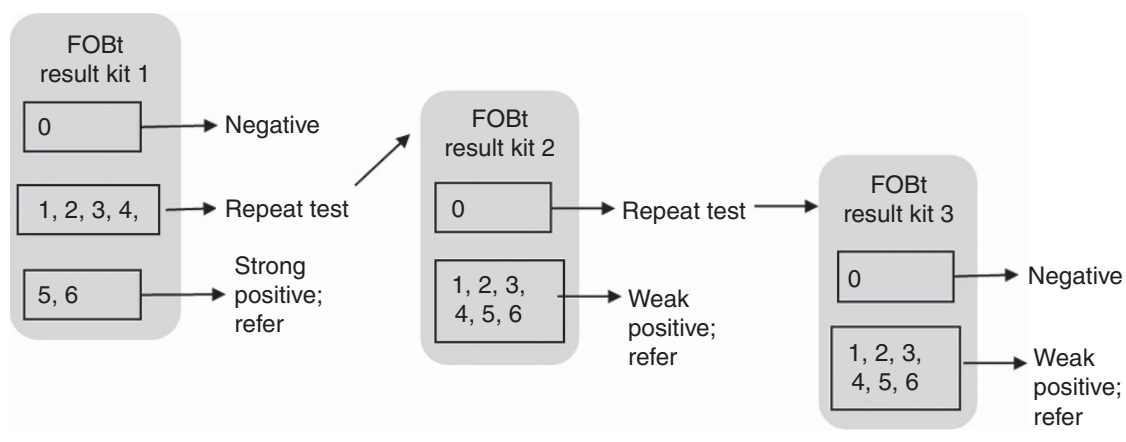

Figure 1. Current referral algorithm for screening with gFOBt. 
The result of each gFOBt kit returned during the screening episode was recorded according to the count of positive spots (0-6). Participants who return a 'normal' first gFOBt (no positive spots) require no further investigation. Participants with 1-6 positive spots on the first gFOBt kit have up to four additional steps along the screening pathway when they could 'drop-out' of screening:

1. Failure to return a second valid gFOBt kit (following an unclear result on the first valid kit).

2. Failure to return a third valid gFOBt kit (following an unclear result on the first valid kit and a normal valid second kit).

3. Failure to attend the SSP clinic (following an abnormal test result [strong positive or a weak positive]).

4. Failure to attend for colonoscopy or other diagnostic test (following attendance at the SSP clinic).

Subjects who return a first gFOBt kit but fail to reach a definitive test result ( 1 and 2 drop-out categories as above) will be invited for screening again in 2 years' time, if they remain within the screening age range. Similarly, subjects who fail to attend the SSP clinic or follow-up investigation (categories 3 and 4 above) will be contacted by the relevant Screening Centre and subjects who remain noncompliant will be returned to routine screening and invited again in 2 years' time if they remain withinin the screening age range. Data on compliance with subsequent screening invitations were not available for this analysis.

Statistical analyses. Data analysis was performed using STATA version 10 (College Station, TX, USA). Separate logistic regression models were built to assess the contribution of age, sex and deprivation to failure to complete screening at each of the four steps along the pathway after 1-6 positive spots on the first kit. Each regression model included participants appropriate for the model; for example, for completion of kit 3, only participants eligible to complete kit 3 (unclear kit 1 and normal kit 2) were included. Tests for linearity and interactions were performed. Further details on the modelling strategy used to derive the final regression model are provided in the Supplementary Material.

The data observed for completion of screening at each of the four steps along the screening pathway were used to predict completion if the threshold for immediate referral to follow-up were reduced from a positive spot count of 5 to just 4 or 3 positive spots. The analysis assumed that completion at each step along the screening pathway was independent of spot positivity (for any kit) (for example that participants with 5-6 positive spots on kit 1 were just as likely to complete a screening episode as participants with 1-2 positive spots on kit 1 ).

\section{RESULTS}

The data set included 1409471 participants aged 60-69 years who were invited to be screened for the first time and had returned at least one valid gFOBt during the study period. One hundred thirty six screening episodes $(0.01 \%$ of the full data set) were excluded because of missing data (details in Supplementary Material). The final data set used for this study represented 1409335 screening episodes.

The majority of participants $(95.08 \%)$ returned a normal first gFOBt kit and were returned to routine screening (Table 1). The following observations were made on the remaining $4.92 \%$ of participants with 1-6 positive spots on the first $\mathrm{gFOBt}$ $(n=69280)$.

In all, 4139 participants (5.97\%) with $1-6$ positive spots on the first gFOBt had either 5 or 6 positive spots and were referred to follow-up. The remaining 65141 participants (94.03\%) had a result that was designated unclear (1-4 positive spots), of whom 94.90\% (61 821) completed a second kit. 27.44\% (16964/ 61821 ) of participants who returned a second gFOBt kit achieved a definitive test result (1-6 spots on kit 2) and the remaining 44857 (72.56\%) participants returned a normal second gFOBt kit and were requested to complete a third kit. Of these, 42770 (95.35\%) completed a third gFOBt kit to achieve a definitive gFOBt result. Overall, $8.30 \%$ (5407/65 141) of participants who had 1 to 4 positive spots on their first gFOBt kit did not complete the required number of gFOBt kits and therefore failed to complete screening (1-4 positive spots on kit 1 but failed to complete kit $2=3320$ (5.10\%); no positive spots on kit 2 and failed to complete kit $3=2087(4.65 \%))$. Almost two-thirds of participants asked to complete more than one gFOBt kit completed three kits to achieve a definitive gFOBt result (42 770/65 141; 65.66\%).

Table 2 shows the proportion of participants referred to an SSP clinic according to the number of positive spots on kit 1 . The majority of participants with 1 or 2 positive spots on the first kit were not referred ( $37446 / 56030 ; 66.83 \%)$, whereas the majority of participants with 3 or 4 positive spots on the first kit were referred $(5,535 / 9,111 ; 60.75 \%)$. All participants with 5 or 6 positive spots were referred immediately; of these 4139 participants $92.10 \%$ attended the SSP clinic appointment, and $80.41 \%$ ultimately complied with the follow-up investigation. Compared with those who were referred immediately, participants who completed multiple kits before referral (24 119) were more likely to both attend the SSP clinic appointment (22969; $95.23 \%$ ), and comply with the follow-up investigation (21029;

Table 1. Distribution of positive spot count results

\begin{tabular}{|c|c|c|c|c|c|c|}
\hline \multirow[b]{2}{*}{$\begin{array}{l}\text { gFOBt-positive } \\
\text { spot count }\end{array}$} & \multicolumn{2}{|c|}{ gFOBt Kit 1} & \multicolumn{2}{|c|}{ gFOBt Kit 2} & \multicolumn{2}{|c|}{ gFOBt Kit 3} \\
\hline & Number & $\%$ & Number & $\%$ & Number & $\%$ \\
\hline 1 & 34805 & 2.47 & 5774 & 9.34 & 3282 & 7.67 \\
\hline 2 & 21225 & 1.51 & 5552 & 8.98 & 2487 & 5.81 \\
\hline 5 & 1798 & 0.13 & 859 & 1.39 & 128 & 0.30 \\
\hline 6 & 2341 & 0.17 & 941 & 1.52 & 136 & 0.32 \\
\hline Total & 1409335 & 100 & 61821 & 100 & 42770 & 100 \\
\hline
\end{tabular}


87.17\%). The higher levels of observed compliance may be because participants had already demonstrated a willingness to comply with the screening pathway by completing multiple kits. Overall, $86.20 \%$ of participants referred for follow-up (24357/28 258) attended the SSP clinic appointment and received a diagnostic test; the remaining $13.80 \%$ of participants failed to complete screening when referred for follow-up after a positive gFOBt.

Data on rates of non-compliance with SSP and diagnostic tests for the demographic variables of age, sex and level of deprivation are provided in the Supplementary Table. There was little variation in compliance with attendance at SSP, whereas the biggest variation in compliance with receiving a diagnostic test was observed for deprivation quintiles, ranging from $92.72 \%$ for the least deprived quintile to $88.69 \%$ for the most deprived quintile.

Among participants receiving a diagnostic test, the proportion with cancer was $19.41 \%(289 / 1489)$ for participants whose gFOBt kit 1 had 5 positive spots and 25.18\% (463/1839) for those with 6 positive spots on kit 1 (Table 2 ).

A detailed flowchart illustrating the observed numbers of participants who completed and did not complete their screening episode is provided in the Supplementary Material.

Factors associated with completion of a screening episode. Results of the logistic regression models are presented in Table 3. Separate regression models were built for men and women because the association between deprivation and attendance at the SSP clinic appeared to be markedly different (see the Supplementary Material for more information).

There was a statistically significant association between age and completion of screening at each of the four steps along the screening pathway after a first gFOBt kit with 1-6 positive spots. Older people were more likely to return a repeat test kit, but were less likely to attend the SSP appointment or the diagnostic investigations. Women were more likely to complete their first repeat test kit, but this association was not observed for subsequent repeat tests or for attending diagnostic investigations. Increasing levels of deprivation were associated with increased odds of not returning either a first or second repeat test kit, as well as increased odds of not attending for a diagnostic investigation. The association between deprivation and attending the SSP clinic was less clear.

The goodness of fit of each model, as measured by the area under the receiver operating characteristic (AUROC) curve, was modest with values ranging between 0.53 and 0.56 .

Possible effect of changing the referral algorithm on completion of screening. The possible effects of changing the spot positivity threshold for requesting a repeat gFOBt kit are presented in Table 4.

The completion rates (under the current algorithm) for persons asked to complete a second gFOBT kit (94.90\%), a third gFOBT kit (95.35\%) and referred for follow-up (86.20\%) were used to predict completion rates for two changes to the algorithm, that is,. referral with three or four positive spots on kit 1 .

A reduction in the threshold for referral to follow-up from five positive spots to four or three positive spots on the first kit inevitably would result in fewer requests for repeat testing and a greater number of people being referred for follow-up. The number of participants failing to complete screening after a request for a repeat gFOBt kit would be reduced, although the number of participants failing to complete screening following referral for

Table 2. Rates of referral and cancer detection by initial gFOBt-positive spot count result

\section{gFOBt kit 1}

positive spot

count
Completed Referred to gFOBt kit

\begin{abstract}
SSP
\end{abstract}
Attended SSP appointment
Received diagnostic test found

Proportion
referred for follow up,
$95 \%$ confidence
interval

Proportion with cancer of those receiving diagnostic tests, 95\% confidence interval

\begin{tabular}{|c|c|c|c|c|c|c|c|c|c|}
\hline 1 & 34805 & 10167 & 9707 & 8945 & 442 & $29.21 \%$ & $28.74-29.69 \%$ & $4.94 \%$ & $4.51-5.41 \%$ \\
\hline 2 & 21225 & 8417 & 8012 & 7344 & 530 & $39.66 \%$ & $39-40.32 \%$ & $7.22 \%$ & $6.65-7.83 \%$ \\
\hline 3 & 4700 & 2794 & 2669 & 2431 & 282 & $59.45 \%$ & $58.04-60.84 \%$ & $11.60 \%$ & $10.39-12.93 \%$ \\
\hline 4 & 4411 & 2741 & 2581 & 2309 & 355 & $62.14 \%$ & $60.7-63.56 \%$ & $15.37 \%$ & $13.96-16.9 \%$ \\
\hline 5 & 1798 & 1798 & 1677 & 1489 & 289 & $100 \%$ & $99.79-100 \%$ & $19.41 \%$ & $17.48-21.5 \%$ \\
\hline 6 & 2341 & 2341 & 2135 & 1839 & 463 & $100 \%$ & $99.84-100 \%$ & $25.18 \%$ & $23.25-27.21 \%$ \\
\hline Total & 69280 & 28258 & 26781 & 24357 & 2361 & $40.79 \%$ & $40.42-41.15 \%$ & $9.69 \%$ & $9.33-10.07 \%$ \\
\hline
\end{tabular}

Abbreviations: $\mathrm{gFOBt}=$ guaiac faecal occult blood test; NHS = National Health Service. Participants aged between 60 and 69 years who returned at least one gFOBt kit in response to their first screening invitation from the NHS Bowel Cancer Screening Southern Programme Hub, where the result of kit 1 was 1-6 positive spots.

Table 3. Predictors of completion at different parts of the screening pathway. See text for definitions

\begin{tabular}{|c|c|c|c|c|c|c|c|c|c|c|}
\hline & \multicolumn{2}{|c|}{ Returning kit 2} & \multicolumn{2}{|c|}{ Returning kit 3} & \multicolumn{2}{|c|}{$\begin{array}{c}\text { Attending } \\
\text { SSP_Females }\end{array}$} & \multicolumn{2}{|r|}{$\begin{array}{l}\text { Attending } \\
\text { SSP_Males }\end{array}$} & \multicolumn{2}{|c|}{$\begin{array}{l}\text { Attending for } \\
\text { investigations }\end{array}$} \\
\hline $\begin{array}{l}\text { Number eligible } \\
\text { Number not } \\
\text { completing (\%) }\end{array}$ & & $\begin{array}{c}65141 \\
3320(5.10)\end{array}$ & & $\begin{array}{c}44857 \\
2087(4.65)\end{array}$ & & $\begin{array}{c}11491 \\
655(5.70)\end{array}$ & & $\begin{array}{c}16767 \\
822(4.90)\end{array}$ & & $\begin{array}{c}26781 \\
2424(9.05)\end{array}$ \\
\hline \multicolumn{11}{|l|}{ Odds ratios } \\
\hline 1 -year increase in age. & 1.013 & (1.002 to 1.025$)$ & 1.050 & \begin{tabular}{|l} 
(1.035 to 1.065$)$ \\
\end{tabular} & 0.975 & (0.952 to 1.000$)$ & 0.970 & (0.949 to 0.992$)$ & 0.975 & (0.962 to 0.988$)$ \\
\hline Female sex & 1.228 & (1.143 to 1.319$)$ & 1.063 & (0.973 to 1.161$)$ & N/A & & N/A & & 0.972 & (0.892 to 1.058$)$ \\
\hline $\begin{array}{l}5 \text {-unit increase in IMD } \\
2007 \text { score }\end{array}$ & 0.929 & (0.915 to 0.943$)$ & 0.937 & (0.919 to 0.954$)$ & 1.038 & (0.998 to 1.078$)$ & 0.997 & (0.966 to 1.029$)$ & 0.923 & (0.907 to 0.939$)$ \\
\hline $\begin{array}{l}\text { Constant; beta } \\
\text { coefficient }\end{array}$ & -2.229 & $(-2.938$ to -1.520$)$ & -0.106 & $(-1.009$ to 0.798$)$ & -4.290 & $(-5.875$ to -2.705$)$ & -4.929 & $(-6.349$ to -3.509$)$ & -4.220 & $(-5.066$ to -3.374$)$ \\
\hline AUROC & 0.556 & (0.546 to 0.566$)$ & 0.558 & (0.546 to 0.571$)$ & 0.539 & (0.517 to 0.562$)$ & 0.527 & (0.506 to 0.547$)$ & 0.553 & (0.541 to 0.565$)$ \\
\hline
\end{tabular}


Table 4. Non-completion at different steps in the screening pathway: observed rates for current BCSP and predicted rates under different thresholds for referral

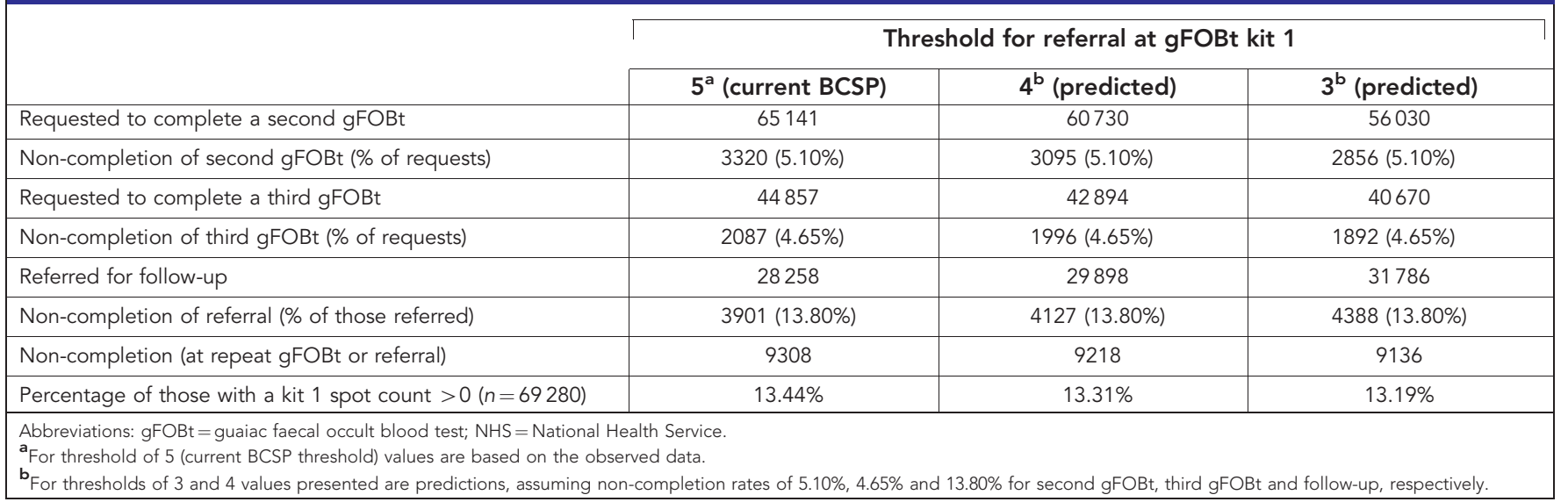

follow-up would increase. The overall increases in participants referred for diagnostic test were estimated to be $5.80 \%$ and $12.49 \%$ for thresholds of four and three positive spots, respectively.

Of the 69280 participants with a spot count greater than zero on their first kit (i.e., participants at risk of failing to complete the screening pathway), overall non-completion (failure to reach a definitive gFOBt result and failure to attend for follow-up if the gFOBt result was abnormal) was $13.44 \%$ using the current threshold for immediate referral (5 positive spots on kit 1). Estimated overall non-completion decreased to $13.31 \%$ for immediate referral with 4 positive spots and to $13.19 \%$ with 3 positive spots, which corresponded to an additional 90 or 172 participants completing the screening pathway. The Southern Hub covers approximately a quarter of the population in England (Bowel Cancer Screening Southern Programme Hub, 2014), so at a national level, approximately 360 or 690 additional people would be expected to complete the screening pathway using referral thresholds of four or three positive spots, respectively.

\section{CONCLUSIONS AND DISCUSSION}

The success of any screening programme depends upon uptake of screening (proportion of invitees achieving a definitive result) and compliance with follow-up investigations for participants with positive primary test results.

We identified four steps in the bowel cancer screening pathway when participants who have returned a first gFOBt kit with 1-6 positive spots may subsequently fail to complete screening. Among participants asked to complete a second gFOBt kit, $5.10 \%$ subsequently failed to complete screening, and $4.65 \%$ of those asked to complete a third gFOBt failed to do so. Among participants who achieved a definitive gFOBt test result and were referred, $13.80 \%$ failed to attend the SSP clinic or a follow-up diagnostic test. Participants with five or six positive spots on kit 1 are more likely to have cancer but only $80.41 \%$ complied with the follow-up investigation. Although some participants may have sought private medical healthcare outside the BCSP, reducing noncompletion (failure to comply with follow-up) in this group should be a priority.

In this data set, 9308 (13.44\%) participants overall returned an initial (valid) gFOBt kit with a non-zero spot count but failed to complete the bowel cancer screening pathway. Cancer was identified in $3.41 \%(2361 / 69280)$ of all participants with 1-6 positive spots on kit 1 and among 9.69\% (2361/24357) of all participants who were referred for follow-up and underwent a diagnostic investigation. This suggests that among the population covered by the Southern Hub and during the six-year study period (October 2006 to October 2012), the number of cancers among participants who failed to complete the screening pathway may have ranged between 317 and 902, assuming that the prevalence of cancer among drop-outs ranged between 3.41 and 9.69\%. As the Southern Hub covers approximately a quarter of the population in England (Bowel Cancer Screening Southern Programme Hub, 2014) and assuming that the rates of non-completion observed in this study are representative of rates nationally, the corresponding number of cancers diagnosed in England could be between 1250 and 3600 over the 6-year period in 60- to 69-year olds screened for the first time. The results of this study suggest that lower thresholds for referral would mean that more participants complete the screening pathway, which will result in more cancers being detected. In addition, lower thresholds would result in more positive results among people who return a first test kit. For a threshold of four (or three) spots positive, this would be people who, under the current screening programme, have four (or three) spots positive on their first kit, followed by no positive spots on kits two and three. These additional positive results would lead to an increase in sensitivity (as there will be more true positive test results), but also a decrease in specificity (as there will be more false positive test results). As estimate of these changes in test characteristics cannot be quantified with the existing data.

Increasing age and having lower levels of deprivation were associated with increased odds for completing a second or third gFOBt kit. Older age and greater deprivation were associated with a significantly decreased odds of attending diagnostic investigations. The association between non-completion of a screening episode, age, sex and deprivation has been studied before (Pornet et al, 2010; Birkenfeld et al, 2011), and the association between poorer uptake and increasing levels of deprivation is well documented (Von Wagner et al, 2009; Birkenfeld et al, 2011; von Wagner et al, 2011). Further, there is some evidence that the requirement to repeat gFOBt kits to reach a definitive test result and failure to attend for follow up is associated with reduced participation in subsequent screening episodes (Lo et al, 2014a; Lo et al, 2014b). However, to the best of our knowledge, this is the first study to examine non-completion at different steps along the screening pathway after returning at least one gFOBt. This is also the first study to explicitly consider potential non-linearity and interactions among the associations between patient demographics and noncompletion of a screening episode.

There are several limitations to this work. The data set was derived from regional data from the BCSP Southern Hub. The South of England has lower levels of deprivation than the rest of 
England (Logan et al, 2012), which may limit the generalisability of the results, although the effect of deprivation was included in the statistical modelling. As an association between levels of deprivation and non-compliance has been demonstrated in this study, the modelled effects of reducing the threshold for referral are likely to be conservative. In addition, only three patient demographics (age, sex and deprivation levels) were available for analyses, and there is a range of other factors that may affect compliance (HoneinAbouHaidar et al, 2014), such as ethnicity (Robb et al, 2008), literacy levels (von Wagner et al, 2009), the presence of certain comorbidities (Heslop et al, 2013) and screening history (Lo et al, 2014a; Lo et al, 2014b). The small number of patient demographics available is likely to be reflected by the relatively low AUROC values (between 0.53 and 0.56) for each of the models. Further, the measure of deprivation employed for this study is area based and so will not be as accurate as individual-based measures of deprivation due to potential heterogeneity in deprivation within an area. The regression results reported here should be interpreted with caution. The true disease status (whether or not a participant has cancer) is not known for all participants. Hence, the impact of a change in the threshold for referral on the number of falsepositive and false-negative test results is unknown. This is likely to be an important consideration for decision makers when considering changes to the threshold, particularly as the impact on numbers completing is modest. Any algorithm changes would also have other changes that are not captured in this analysis. This includes the additional resources and costs required for additional colonoscopies, and the potential impact on participants' quality of life. The assumption that completion of a screening episode is not dependent upon the number of spots positive on any gFOBt kit and that the data observed for the current algorithm can be used to predict completion of a screening episode if the referral were changed, requires further investigation. The restriction to first screening episodes may also limit the generalisability of the findings to current screening programmes. However, uptake rates are much higher for subsequent screens than for initial screens (Kearns et al, 2014), which suggest that attempts to improve completion should focus on initial screening episodes. A final important limitation to note is that not all of the participants who fail to complete the screening pathway will do so for reasons that are amenable to intervention. For example, participants may leave the pathway for clinical reasons, or may choose to change to a private healthcare provider. These reasons are most likely to occur for participants who fail to attend for further diagnostic investigations, and may explain why rates of non-completion are highest at this step in the screening pathway. Hence, the noncompletion rates reported here could be overestimated.

Within the BCSP, once-only screening with flexible sigmoidoscopy at age 55 years is being introduced in addition to screening with gFOBt for 60- to 74-year olds (Geurts et al, 2015), whereas the use of faecal immunochemical testing for haemoglobin (FIT) instead of the gFOBT has been piloted in the south of England (Bowel Cancer Screening Southern Programme Hub, 2015; Moss et al, 2015). These changes may have an impact on the completion of screening episodes. However, in order to assess the effectiveness of these changes, comparisons shall be required with the existing evidence about the sociodemographic factors that are associated with drop-out, and how these vary across the current screening programme. In addition, many other countries use gFOBt screening (Benson et al, 2012), and so the results presented here will be of interest beyond the BCSP.

As with interventions to increase screening uptake (Shankaran et al, 2007), targeted interventions may be required to help tackle potential health inequalities (Asaria et al, 2014). On the basis of the results of this study, and noting the previously mentioned limitations, it is recommended that measures to improve completion should be targeted at participants who are referred for further investigations, with particular emphasis on older participants and those from more deprived areas. As an increasing level of deprivation is associated with a reduction in both uptake and completion, any interventions aimed at improving uptake among these areas may also have a beneficial effect on compliance. Further research eliciting the reasons for non-completion at each part of the screening pathway would also be beneficial.

Changing the spot positivity threshold for repeating gFOBt is likely to have several effects (Geraghty et al, 2014). There would be a reduction in the number of participants being asked to provide more than one gFOBt kit and an increase in endoscopy requirements due to an increased number of referrals. Such a change is likely to improve completion at repeat testing but decrease completion at referral. The combination of these two effects may improve overall completion of screening episodes, although the effect is likely to be small. Larger variations in uptake were observed by deprivation quintile, which may require more urgent attention.

Failure by participants to complete the bowel cancer screening pathway will result in some cancers not being identified. Targeted measures to improve completion of the screening pathway, with a particular emphasis on participants who are referred for follow-up and more deprived groups, could help to improve outcomes within the BCSP.

\section{CONFLICT OF INTEREST}

The authors declare no conflict of interest.

\section{REFERENCES}

Altobelli E, Lattanzi A, Paduano R, Varassi G, di Orio F (2014) Colorectal cancer prevention in Europe: burden of disease and status of screening programs. Prev Med 62: 132-141.

Asaria M, Griffin S, Cookson R, Whyte S, Tappenden P (2014)

Distributional Cost-Effectiveness Analysis of Health Care ProgrammesA Methodological Case Study of the UK Bowel Cancer Screening Programme. Health Econ 24(6): 742-754.

Aubin-Auger I, Mercier A, Lebeau JP, Baumann L, Peremans L, Van Royen P (2011) Obstacles to colorectal screening in general practice: a qualitative study of GPs and patients. Fam Pract 28(6): 670-676.

Benson VS, Atkin WS, Green J, Nadel MR, Patnick J, Smith RA, Villain P (2012) Toward standardizing and reporting colorectal cancer screening indicators on an international level: the International Colorectal Cancer Screening Network. Int J Cancer 130(12): 2961-2973.

Beydoun HA, Beydoun MA (2008) Predictors of colorectal cancer screening behaviors among average-risk older adults in the United States. Cancer Causes Control 19(4): 339-359.

Birkenfeld S, Belfer RG, Chared M, Vilkin A, Barchana M, Lifshitz I, Fruchter D, Aronski D, Balicer R, Niv Y (2011) Factors affecting compliance in faecal occult blood testing: a cluster randomized study of the faecal immunochemical test versus the guaiac faecal occult test. J Med Screen 18(3): 135-141.

Bowel Cancer Screening Southern Programme Hub (2014) Bowel Cancer Screening Southern Programme Hub-Summary report: 2012-2013. Available at http://royalsurrey.staging.flipsidegroup.com/bowel-cancerscreening-southern-hub (accessed 28 December 2015).

Bowel Cancer Screening Southern Programme Hub (2015) Bowel Cancer Screening Southern Programme Hub-Summary report: 2013-2014. Available at http://royalsurrey.staging.flipsidegroup.com/bowel-cancerscreening-southern-hub (accessed 28 December 2015).

Brouse CH, Basch CE, Wolf RL, Shmukler C, Neugut AI, Shea S (2003) Barriers to colorectal cancer screening with fecal occult blood testing in a predominantly minority urban population: a qualitative study. Am J Public Health 93(8): 1268-1271.

Cancer Research UK (2013) Cancer Statistics: Key Facts: Bowel Cancer April 2013. 
Communities and Neigbourhoods (2010) Indices of Deprivation 2007. Department for Communities and Local Government.

Fraser CG, Digby J, McDonald PJ, Strachan JA, Carey FA, Steele RJ (2012) Experience with a two-tier reflex gFOBT/FIT strategy in a national bowel screening programme. J Med Screen 19(1): 8-13.

Geraghty J, Butler P, Seaman H, Snowball J, Sarkar S, Blanks R, Halloran S, Bodger K, Rees C (2014) Optimising faecal occult blood screening: retrospective analysis of NHS Bowel Cancer Screening data to improve the screening algorithm. Br J Cancer 111(11): 2156-2162.

Geurts SME, Massat NJ, Duffy SW (2015) Likely effect of adding flexible sigmoidoscopy to the English NHS Bowel Cancer Screening Programme: impact on colorectal cancer cases and deaths. Br J Cancer 113(1): 142-149.

Gupta S (2008) Will test-specific adherence predict the best colorectal cancer screening strategy? Ann Intern Med 149(9): 659-669.

Hardcastle JD, Chamberlain JO, Robinson MH, Moss SM, Amar SS, Balfour TW, James PD, Mangham CM (1996) Randomised controlled trial of faecal-occult-blood screening for colorectal cancer. Lancet 348(9040): 1472-1477.

Heslop P, Blair P, Fleming P, Hoghton M, Marriott A, Russ L (2013) Confidential inquiry into premature deaths of people with learning disabilities (CIPOLD): final report. Available at www.bristol.ac.uk/cipold/ fullfinalreport.pdf (accessed 28 December 2015).

Hewitson P, Glasziou P, Watson E, Towler B, Irwig L (2008) Cochrane systematic review of colorectal cancer screening using the fecal occult blood test (hemoccult): an update. Am J Gastroenterol 103(6): 1541-1549.

Honein-AbouHaidar GN, Kastner M, Vuong V, Perrier L, Rabeneck L, Tinmouth J, Straus S, Baxter NN (2014) Benefits and barriers to participation in colorectal cancer screening: a protocol for a systematic review and synthesis of qualitative studies. BMJ Open 4(2): e004508.

Kearns B, Whyte S, Chilcott J, Patnick J (2014) Guaiac faecal occult blood test performance at initial and repeat screens in the English Bowel Cancer Screening Programme. Br J Cancer 111(9): 1734-1741.

Kewenter J, Brevinge H, Engaras B, Haglind E, Ahran C (1994) Results of screening, rescreening, and follow-up in a prospective randomized study for detection of colorectal cancer by fecal occult blood testing: results for 68,308 subjects. Scand J Gastroenterol 29(5): 468-473.

Kronborg O, Fenger C, Olsen JA, Jargensen OD, Sandergaard O (1996) Randomised study of screening for colorectal cancer with faecal-occultblood test. Lancet 348(9040): 1467-1471.

Lo SH, Halloran S, Snowball J, Seaman H, Wardle J, Von Wagner C (2014a) Predictors of repeat participation in the NHS bowel cancer screening programme. Br J Cancer 112(1): 199-206.

Lo SH, Halloran S, Snowball J, Seaman H, Wardle J, von Wagner C (2014b) Colorectal cancer screening uptake over three biennial invitation rounds in the English bowel cancer screening programme. Gut 64(2): 282-291.

Logan RF, Patnick J, Nickerson C, Coleman L, Rutter MD, von Wagner C (2012) Outcomes of the Bowel Cancer Screening Programme (BCSP) in England after the first 1 million tests. Gut 61(10): 1439-1446.

Mandel JS, Bond JH, Church TR, Snover DC, Bradley GM, Schuman LM, Ederer F (1993) Reducing mortality from colorectal cancer by screening for fecal occult blood. N Engl J Med 328(19): 1365-1371.

Moss S, Mathews C, Day T, Smith S, Halloran SP (2015) OC-002 Increased participation in colorectal cancer screening during a pilot of faecal immunochemical test for haemoglobin (FIT) in england. Gut 64(Suppl 1): A1.

Moss SM, Campbell C, Melia J, Coleman D, Smith S, Parker R, Ramsell P, Patnick J, Weller DP (2012) Performance measures in three rounds of the English bowel cancer screening pilot. Gut 61(1): 101-107.

NHS Bowel Cancer Screening Programme (2011) In the loop newsletter for people working in the NHS Bowel Cancer Screening Programme, Winter 2010/2011, pp 1-4.

Pornet C, Dejardin O, Morlais F, Bouvier VÃ, Launoy G (2010) Socioeconomic determinants for compliance to colorectal cancer screening. A multilevel analysis. J Epidemiol Community Health 64(4): 318-324.

Robb KA, Power E, Atkin W, Wardle J (2008) Ethnic differences in participation in flexible sigmoidoscopy screening in the UK. J Med Screen 15(3): 130-136.

Robinson MHE, Moss SM, Hardcastle JD, Whynes DK, Chamberlain JO, Mangham CM (1995) Effect of retesting with dietary restriction in Haemoccult screening for colorectal cancer. J Med Screen 2(1): $41-44$.

Shankaran V, McKoy JM, Dandade N, Nonzee N, Tigue CA, Bennett CL, Denberg TD (2007) Costs and cost-effectiveness of a low-intensity patientdirected intervention to promote colorectal cancer screening. J Clin Oncol 25(33): 5248-5253.

Sicsic J, Franc C (2014) Obstacles to the uptake of breast, cervical, and colorectal cancer screenings: what remains to be achieved by French national programmes? BMC Health Serv Res 14(1): 465.

Smith SK, Simpson JM, Trevena LJ, McCaffery KJ (2014) Factors associated with informed decisions and participation in bowel cancer screening among adults with lower education and literacy. Med Decis Making 34(6): 756-772.

UK Colorectal Cancer Screening Pilot Group (2004) Results of the first round of a demonstration pilot of screening for colorectal cancer in the United Kingdom. BMJ 329(7458): 133.

Von Wagner C, Good A, Wright D, Rachet B, Obichere A, Bloom S, Wardle J (2009) Inequalities in colorectal cancer screening participation in the first round of the national screening programme in England. Br J Cancer 101: S60-S63.

von Wagner C, Baio G, Raine R, Snowball J, Morris S, Atkin W, Obichere A, Handley G, Logan RF, Rainbow S (2011) Inequalities in participation in an organized national colorectal cancer screening programme: results from the first 2.6 million invitations in England. Int J Epidemiol 40(3): 712-718.

von Wagner C, Semmler C, Good A, Wardle J (2009) Health literacy and self-efficacy for participating in colorectal cancer screening: the role of information processing. Patient Educ Couns 75(3): 352-357.

Weller DP, Campbell C (2009) Uptake in cancer screening programmes: a priority in cancer control. Br J Cancer 101: S55-S59.

This work is published under the standard license to publish agreement. After 12 months the work will become freely available and the license terms will switch to a Creative Commons AttributionNonCommercial-Share Alike 4.0 Unported License.

Supplementary Information accompanies this paper on British Journal of Cancer website (http://www.nature.com/bjc) 